\title{
Role of Toll like Receptor(s) in Tumor Biology
}

\author{
Sweta Sanguri and Damodar Gupta* \\ Division of Metabolic Cell Signaling Research, Institute of Nuclear Medicine \& Allied Sciences, India
}

Submission: March 10, 2017; Published: April 24, 2017

*Corresponding author: Damodar Gupta, Division of Metabolic Cell Signaling Research, Institute of Nuclear Medicine \& Allied Sciences, Brig. SK Mazumdar Marg, Defence Research and Development Organization, Ministry of Defence, Delhi 110054, India, Email: damodar.gupta@gmail.com

\begin{abstract}
Toll like receptors belong to a family of specialized immune receptors that highly conserved and specific for their ligands. TLR signaling can identify specific pathogen and can induce expression of inflammatory cytokines, chemokines, initiate opsonization and phagocytosis, elicit reactive oxygen/nitrogen species production, activate the complement pathway etc. to get rid of it. Apart from its unquestionable role in pathogen recognition and neutralization, TLRs can also identify altered self-proteins including those expressed by transformed cell. Agonists of toll like receptors, both of natural or synthetic origin have been exploited for their ability to elicit and/or potentiate anticancer response alone or when combined with available treatment regimes. TLR agonist mediated activation of immune cells (lymphocytes, leukocytes etc.) and production of inflammatory mediators leads to the generation of cellular (adaptive) immune responses that hold promising applications against cancer progression. TLRs are expressed in cells of immune system including dendritic cells (DC) and macrophages, natural killer cells, cells of the adaptive immunity ( $\mathrm{T}$ and B lymphocytes), and epithelial, endothelial, fibroblasts of different tissues. Tumor cells also express TLR, however, differential TLR expression in different cancer cell type leads to conflicting response(s) after activation with TLR agonists, which can be protumor or antitumor. TLR mediated inflammatory response(s), anti-apoptotic potential and epithelial regeneration properties may promote carcinogenesis. Moreover, several mechanisms including the induction of $\mathrm{eT}_{\text {regs }}$ and abrogation of CTLs help the tumors evade cancer immune-surveillance. Therefore, a TLR agonist need to be selected for cancer therapy, based on the tumor microenvironment and a rigorous calculation of effects on immune cells, including $\mathrm{T}$ cell population (enhance effector and memory CTL activity, reduce $\mathrm{eT}_{\text {reg }}$ function).
\end{abstract}

Keywords: Toll Like receptors; TLR agonists; Cancer immunotherapy; TLR signaling; Tumor biology

Abbreviations: TLR: Toll Like Receptors; PRR: Pattern Recognition Receptors; IL: Interleukins; PAMP: Pathogen-Associated Molecular Patterns; APC: Antigen Presenting Cells; DC: Dendritic Cells; CTL: Cytotoxic T Lymphocyte; $\mathrm{eT}_{\text {regs }}$ : Effector T Regulatory cells; OV: Oncolytic Virus

\section{Introduction}

TLRs belong to the pattern recognition receptors (PRR) family that is known to sense both self and non-self danger signals [1]. They are crucial and important components of innate immune system, and act as first line of defence against different potential pathogens [2]. TLRs recognize broad classes of molecular structures including the lipopeptides, lipoteichoic acids, lipopolysaccharides, peptidoglycans, zymogens, mannans, flagellins, viral and bacterial nucleic acids etc. They initiate signal transduction cascades resulting in induction of inflammatory cytokines like the interferons, the interleukinsIL2, IL6, IL8, IL12, IL16, and TNF- $\alpha$ (as one of the responses) to get rid of unwanted organisms, pathogens and transformed selftissues to maintain cellular homeostasis [3,4]. In addition, TLR signaling can trigger apoptosis, opsonization and phagocytosis, induction of reactive oxygen/nitrogen species, activation of the complement pathway etc. depending on the ligand (pathogen) sensed [5]. Furthermore, activation of TLRs is also known to activate intracellular signaling pathways resulting in activation of transcription factors including NF $\mathrm{KB}, \mathrm{MAPK}$ etc. NF- $\kappa \mathrm{B}$ regulates expression of anti-apoptotic genes, cytokines and growth factors [6]. Eleven different TLRs have been identified in humans till date and the TLR signaling is well studied and documented [7]. Various TLRs identified till date are summarized in Table 1 [812]. TLRs also play an important role in the initiation of adaptive immune responses [13]. Pathogen (ligand) specific adaptive immunity develops due to activation of antigen presentation, TLR-dependent expression of pro inflammatory cytokines and up regulation of co-stimulatory molecules [14]. Moreover, T lymphocytes also express selective TLRs and can be directly activated by their respective ligands [15]. Toll-like receptors have also been shown to be an important link between innate and adaptive immunity $[5,13,14]$.

TLR signaling plays differential role in maintenance of cellular homeostasis and the results include cell proliferation or 
apoptosis, cell differentiation, as well as induction of different chemokines, inflammatory cytokines including interleukins and interferons $[16,17]$. Both the innate and acquired immune system has precise role in rendering protection to organism from foreign pathogens and altered self-tissues while sustaining immunetolerance to normal self-tissues. TLRs play twin, but contrasting role in tumor biology. They are capable of maintaining antitumor

\section{TLR Signaling}

Table 1: TLR ligands and roles in cancer progression.

\begin{tabular}{|c|c|c|c|}
\hline TLRs & Ligands & $\begin{array}{l}\text { Association with Tumor } \\
\text { Biology/Response(S) }\end{array}$ & Reference \\
\hline TLR 1 & $\begin{array}{l}\text { Cooperate with TLR2 against } \\
\text { triacyllipopeptides }\end{array}$ & Tumor Regression & {$[27,31,42]$} \\
\hline TLR 2 & $\begin{array}{c}\text { Lipopeptide, peptidoglycan, and } \\
\text { lipoteichoic acid }\end{array}$ & $\begin{array}{l}\text { Reduces tumor progression and } \\
\text { prolongs survival }\end{array}$ & {$[27,33,8]$} \\
\hline TLR 3 & $\begin{array}{c}\text { Double-stranded RNA, } \\
\text { Polyinosine-polycytidylic acid }\end{array}$ & $\begin{array}{l}\text { Reduces Lung cancer, } \\
\text { Hepatocellular carcinoma }\end{array}$ & {$[2,20]$} \\
\hline TLR 4 & LPS, HMGB1 & $\begin{array}{c}\text { Promotes (Hepatocellular } \\
\text { carcinoma and cervical cancer) } \\
\text { and reduces tumor progression } \\
\text { and prolongs survival }\end{array}$ & {$[14,32,33,20]$} \\
\hline TLR 5 & Flagellin & $\begin{array}{c}\text { Promotes (gastric) cancer or } \\
\text { suppress (CBLB } 502 \text { breast and } \\
\text { lung, head and neck, colon) cancer }\end{array}$ & {$[18,9,10]$} \\
\hline TLR 6 & $\begin{array}{l}\text { Cooperate with TLR2 against } \\
\text { diacyllipopeptides }\end{array}$ & Tumor Regression & [27] \\
\hline TLR 7 & ssRNA & $\begin{array}{l}\text { Metastasis (Lung cancer), } \\
\text { antitumor }\end{array}$ & {$[11,28,42]$} \\
\hline TLR 8 & ssRNA & $\begin{array}{l}\text { Metastasis (Lung cancer), } \\
\text { antitumor }\end{array}$ & {$[11,12]$} \\
\hline TLR 9 & $\mathrm{CpG}$ & $\begin{array}{l}\text { Metastasis (Breast, gastric, } \\
\text { ovarian cancer glioma), antitumor }\end{array}$ & {$[11,20]$} \\
\hline TLR 10 & Unknown natural ligand & - & \\
\hline TLR 11 & Expression in mouse cells & Toxoplasma gondiiprofilin & {$[20]$} \\
\hline TLR 12 & Expression in mouse cells & Unknown & \\
\hline TLR 13 & Expression in mouse cells & Viral & {$[20]$} \\
\hline
\end{tabular}

TLR: Toll like Receptor; HMGB1: High Mobility Group Box 1 Protein; LPS: Lipo Poly Saccharide

TLRs are trans membrane proteins that trigger the signal transduction cascades after interaction with specific pathogenassociated molecular patterns (PAMP) ligands and play a critical role in innate and adaptive immune responses [5]. TLR1, TLR2, TLR4, TLR5, TLR6, and TLR11 are located on the cell surface and TLR3, TLR7, TLR8, and TLR9 are localized to the endosomal/ lysosomal compartment [21]. The cellular distribution patterns of TLR is complex; TLRs are expressed on the membranes of dendritic cells, macrophages, natural killer cells, cells of the adaptive immunity ( $\mathrm{T}$ and $\mathrm{B}$ lymphocytes) and non-immune cells (epithelial, endothelial cells, and fibroblasts). TLR1, predominantly distributed in monocytes/macrophages and PMNs. TLR2 can be found on professional immune cells, including neutrophils, macrophages, and dendritic cells, but also on stromal cells throughout the body. In human naive B cells, most of the TLRs are expressed at low to undetectable levels, but TLR9 and environment by eliciting activation of anti-tumor mediators such as type I interferon [18]. Remarkably, TLRs are even documented to play supportive role for initiation, progression and metastasis of cancer [19,20]. Since, transformed tissues are altered selfderived tissue, and therefore sometimes can evade immunesurveillance. In the present review, we briefly review role of TLR signaling in tumor biology. 
TLRs activate the conserved MyD88-dependent pathway leading to IKK complex and AP-1 activation, which are responsible for inflammatory responses. TLRs recruits differential adaptor leading to variations in the expression of downstream signaling effectors [16,21]. An overview of TLR signaling is briefly illustrated in Figure 1.

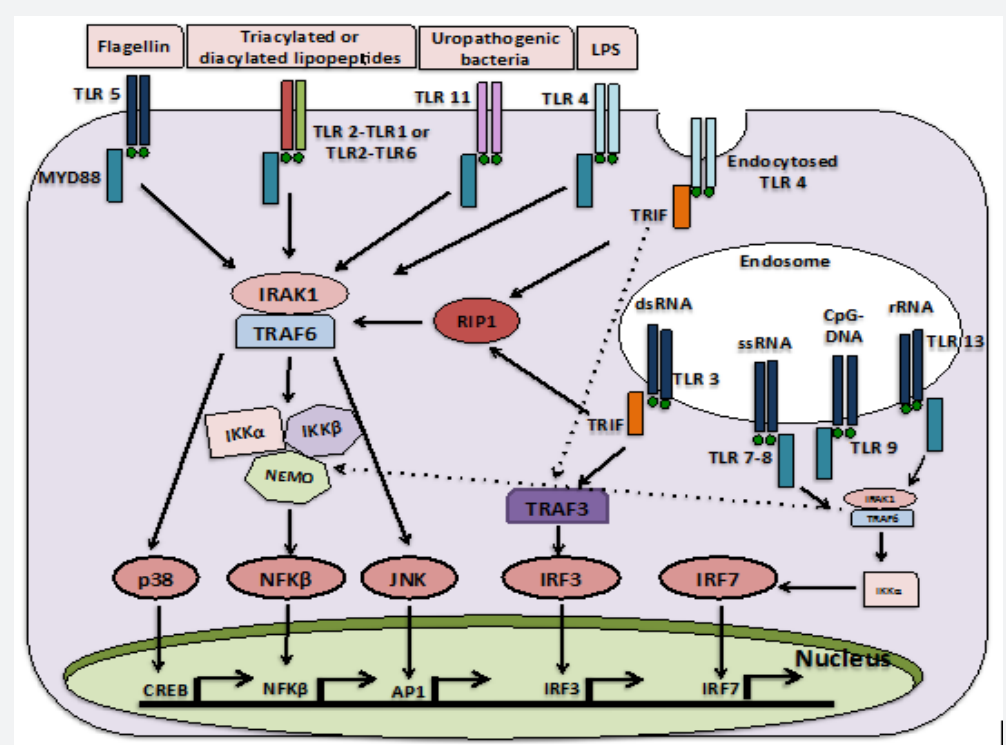

Figure 1: Toll like receptor signaling pathways Toll-like receptors (TLRs) recognize distinct pathogen-associated molecular patterns and play a critical role in innate immune responses. 11 members of the TLR family have been identified till date. The activation of the TLR signaling pathway originates from the cytoplasmic TIR domain that associates with an adaptor, MyD88. IRAK-1 is activated by phosphorylation and associates with TRAF6, thereby activating the IKK complex and leading to activation of MAP kinases (JNK, p38 MAPK) and NF-KB. Activation of MyD88- independent pathways occurs via TRIF and TRAF3. TLRs also mediate alternative pathways by utilizing a specific combination of adapter molecules to mount appropriate immune responses [7].

TLR Mediated Functions in Innate and Acquired Immunity

TLRs detect the presence of invading pathogen, recognize pathogen type and elicit an immediate pathogen specific response [7]. TLRs present in dendritic cells (DC) and macrophages persistently detect non-self molecular patterns (pathogen) from the external environment viz. skin and mucosal surfaces [13]. Cells of peripheral blood and lymph including natural killer cells, cells of the adaptive immunity ( $\mathrm{T}$ and B lymphocytes) etc. constantly explore the internal environment. Epithelial, endothelial cells, and fibroblasts also express TLRs and sense invasion of pathogens [15]. Recognition of specific pattern of invading pathogen by TLR is associated with instantaneous production of specific pro-inflammatory cytokines and chemokines. TLR signaling induces chemotactic molecules and initiate recruitment specific leukocyte (neutrophils, macrophages, and NK cells) with unique effector functions (reactive oxygen radicals, antimicrobial peptides, phagocytosis and cytokines, and perforin-mediated killing) [22]. Consequently, innate responses have the potential for causing significant tissue damage, locally and systemically and can lead to acute or chronic inflammatory disease states [23].

Toll-like receptors have also been shown to be an important interface between the two types of immune responses $[5,13,14]$. Antigen presenting cells, primarily dendritic cells are important connecting link between innate and adaptive immunity. Presence of TLR ligands induces TLR-dependent gene expression of inflammatory cytokines and co-stimulatory molecules leading to maturation of immature DC, T helper or cytotoxic T cells subset activation and differentiation $[5,14,16]$. B and $\mathrm{T}$ lymphocytes themselves express TLRs and can be directly activated by their respective ligands [15]. TLRs stimulate adaptive response with effector functions appropriate to the type of pathogen. Presence of different set of TLR decides cellular response to pathogen exposure and TLR agonists can produce strikingly different responses when administered by different routes.

\section{TLR Agonists: Role in Cancer Progression}

Local irritation and chronic inflammation enhances tumor development and progression, and is known to promote survival, proliferation and migration of cancer cells [22]. TLR mediated inflammation; anti-apoptotic and wound healing (epithelial regeneration and myofibroblast activation) properties may together or independently promote carcinogenesis [24]. The inflammatory process is initiated in order to heal the affected tissue. It has been shown that TLR2 (4q32) and TLR4 (9q33.1) genes are highly polymorphic in nature [25]. The polymorphism causes differential inflammatory response by changes in protein expression or function and can influence the progression of several cancer types. Intestinal tissue is in contact to several microorganisms and in a condition of heightened inflammatory response. Polymorphism of genes involved in the inflammatory and immune responses may contribute to an increased risk of 
developing cancer. TLRs are expressed in macrophages and other myeloid cells, which are found in solid tumors [23]. M2 macrophages along with myeloid-derived suppressor cells have been documented to contribute in immune suppression in the tumor microenvironment. TLRs influence expression of IL-4, IL-13, IL-10 and M-CSF that induce tumor-promoting M2 macrophages and reduce cytotoxicity of M1 macrophages. Both these events can contribute to immune evasion of tumor cells. Effector $\mathrm{T}_{\text {regs }}\left(\mathrm{eT}_{\text {regs }}\right)$ with a CD45RA-Foxp $3^{++} \mathrm{CCR} 4^{+}$ phenotype are the regulatory $\mathrm{T}\left(\mathrm{T}_{\text {reg }}\right)$ cells with the most potent immunosuppressive activity [26]. $\mathrm{eT}_{\text {regs }}$ counteract the CTL responses either through cell contact or by secreting suppressive cytokines. TLR4 and TLR5 are known to directly enhance $\mathrm{eT}_{\text {reg }}$ function; TLR8 signals abolish their suppressive activities. A synthetic bacterial lipoprotein (BLP), has been shown to effectively abrogate $\mathrm{eT}_{\text {reg }}$ function [27]. TLR1/2 agonists have been proposed to be exceptional candidates for cancer immunotherapy clinical trials. TLR ligands and roles in cancer biology have been briefly illustrated in Table 1. US-FDA and equivalent agencies worldwide approved TLR agonist has been summarized in Figure 2.

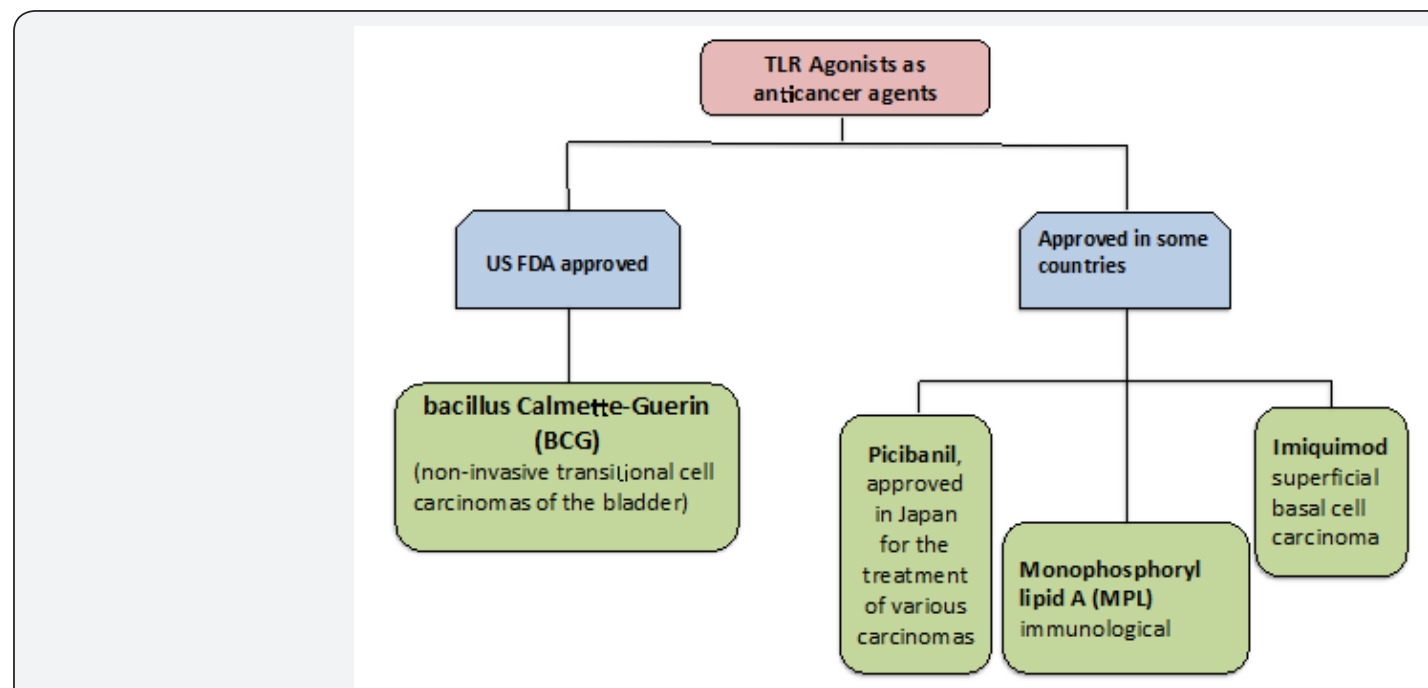

Figure 2: US-FDA and equivalent agencies worldwide approved TLR agonists for use in humans for antineoplastic effects as standalone (BCG) or combination therapeutic intervention. BCG is an attenuated variant of Mycobacterium bovis (TLR2/TLR4 agonist), Picibanil (OK432) is a lyophilized preparation of Streptococcus pyogenes (a mixed TLR2/TLR4 agonist), monophosphoryl lipid A (MPL) is a derivative of Salmonella minnesota LPS employed as immunological adjuvant, imiquimod (TLR7 agonist) is an imidazoquinoline derivative [23,37].

\section{TLR Agonists: Role in Anti Tumor Therapy}

Cytotoxic T lymphocytes play a pivotal role in tumor immune surveillance by specifically recognizing and killing tumor cells and maintaining tumor-specific immune memory [23]. However, cancer cells are self-derived and generally poor immunogens; can bypass immuno-surveillance by lack of antigen-specific $\mathrm{T}$ cell responses [22]. A variety of mechanisms including the induction of $\mathrm{eT}_{\text {regs }}$ help the tumors evade the elimination and equilibrium phases of cancer immuno-editing [20,22]. TLR agonists are identified as powerful immune adjuvants for antigen-presenting cells (APC) activation. Activation of APCs through TLRs has been exploited by numerous traditional chemotherapeutic drugs that trigger innate and adaptive immune activation [23]. Bacterial lipoprotein (BLP) has been shown to mediate tumor regression by abolishing $\mathrm{eT}_{\text {regs }}$ function [27]. TLR agonists trigger rapid and coordinated modulation of immune response including activation of immune cells (lymphocytes, leukocytes etc.), production of inflammatory mediators, chemokines, cytokines that leads to the generation of cellular (adaptive) immune responses [28]. These events hold promising applications for the treatment of inflammation and cancer [29,30]. An ideal therapeutic TLR agonist has been proposed to enhance effector and memory CTL activity, reduce $\mathrm{eT}_{\text {reg }}$ function and not encourage tumorigenesis [27]. Several TLR agonists are currently being investigated for their antitumor efficacy and are currently under different stages of drug development [31]. However, BCG is the only US-FDA approved TLR agonist as a standalone therapeutic intervention. TLR agonists are increasingly explored for their ability to enhance immune responses against tumors and infectious agents [32]. They have an ability to stimulate APCs resulting in activation of tumor-specific $\mathrm{T}$ cell responses [20]. Thus, selective down regulation of $\mathrm{eT}_{\text {reg }}$ function, coupled with the promotion of cytotoxic T lymphocyte (CTL) activity, may represent an attractive strategy for future cancer immunotherapy [27]. TLR2/4 agonists have been shown to induce TNF- $\alpha$ secretion, inducible nitric oxide synthase expression, activate dendritic cell traffic and cytotoxic T-cell responses [33]. US-FDA and equivalent agencies worldwide approved TLR agonists for use in humans against cancer have been summarized in Figure 2 [28].

\section{Synergistic Application of TLR Agonist and Other Cancer Therapies}

Radiation therapy is a conventional antitumor strategy that kills cancer cells by damaging their DNA directly or indirectly, 
through generation of ROS. Damage to the DNA, can elicit activation of DNA repair pathway or cell death pathway based on the extent of damage and chances of DNA repair. Exposure of living cells to ionizing radiation can result in several biological effects that can be acute or chronic, somatic or hereditary, stochastic or deterministic in nature $[34,35]$. Radiation mediated ROS causes extensive damage to important biomolecules including lipids, phospholipids along with protein components $[36,37]$. ROS mediated oxidation of biomolecules compromises structure, integrity and biological function of important cell organelle ultimately driving cell to undergo mitotic catastrophe or cell death [38]. Conversely, radiation therapy also causes extensive damage to normal cells and research is directed towards identification of efficient radio sensitizers and radio protectors that would decrease the clinical dosage of radiation [28]. Focused damage to cancer cells by synergistic application of TLR or combination of different TLR agonists have been increasingly explored and shown to possess protective efficacy in normal cells, include a synthethic flagellin, CBLB502TLR 5 agonist $[39,40]$. CpG ODN, a TLR9 agonist in combination with radiotherapy has also been shown as a promising strategy possessing potent immuno stimulatory effect [41].

TLR agonists along with combined treatment of chemotherapeutics to tumor cells has also been extensively explored to get higher efficacy of antitumor effect compared in lesser clinical dosage of chemotherapeutics [20,42]. Activation of different TLRs plays opposing roles, both antitumor or protumor based on the activation of TLRs on immune cells (or cancer cells themselves), induction of differential cytokines or/ costimulatory molecules on APCs (or tumor cells), effects on T cell proliferation and differentiation etc $[20,26]$. Motolimod, is a toll-like receptor 8 (TLR8) agonist currently in multiple clinical trials in combination with chemotherapeutics and monoclonal antibodies. It has been shown to mobilize a patient's own immune system to stimulate an immune response in the tumor microenvironment [29]. The activation of myeloid dendritic cells by motolimod leads to an immune cascade that recruits other immune cells including NK cells and T lymphocytes that enhance tumor cell killing [29,30].

Oncolytic virus (OVs) causes selective tumor cell lysis and induces immunogenic (necrosis, pyroptosis and autophagic) cell death. The lysed tumor cells release cellular proteins, that acts as DAMP and initiate innate and adaptive immune response(s). TLR agonists have also been shown to possess promising potential in potentiating anticancer cancer response when combined with oncolytic viral (OV) therapy [43]. TLR agonists are also currently investigated as vaccine adjuvants in anticancer therapies for their ability to activate immune cells and promote inflammation. Agonists of TLR2 (BLP), TLR3 (Poly I: C), TLR4 (MPL), TLR7 (resiquimod and imiquimod) and TLR9 (CpG ODNs) have been shown as potent cancer vaccine adjuvants and as immunotherapeutic agents [31,44].

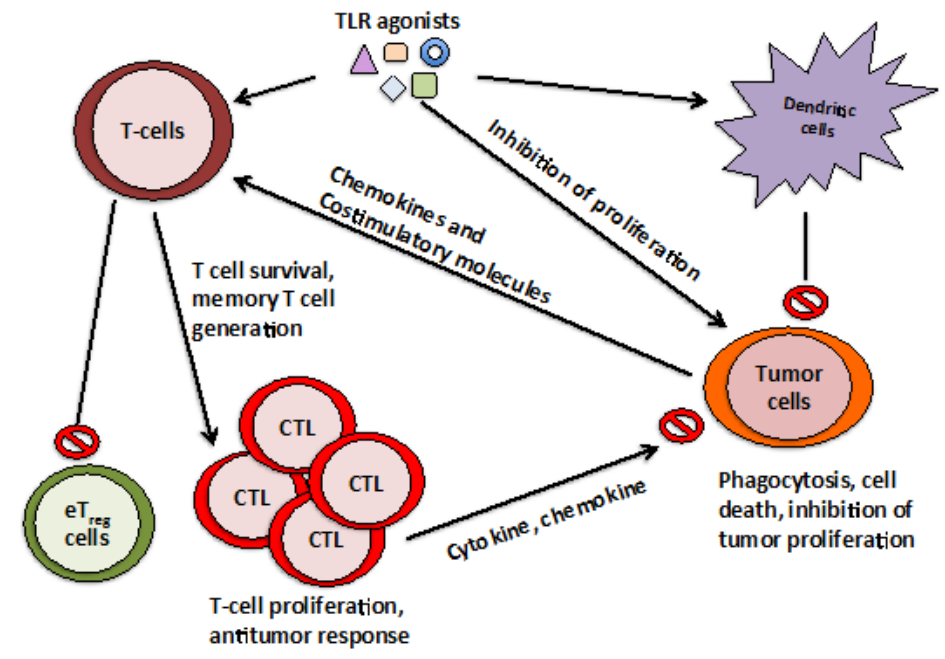

Figure 3: TLR agonists as an antitumor agentto induce potent anticancer responses. The TLR agonists should kill tumor cells directly and/ or prevent tumor proliferation. It should activate APCs, inhibit $\mathrm{eT}_{\text {Reg }}$ cells, induce proliferation of CTL and increase cytotoxicity of tumor cells [15].

\section{Discussion and Conclusion}

Decreased propensity of altered cells for apoptosis contributes to tumor formation and immune evasion helps in its progression. The present commodities against cancer include chemotherapy, radiotherapy and immunotherapy. Besides, narrow therapeutic index and intense side effects to normal cells other phenomenon exhibited commonly include tumor relapse, and tumor drug resistance. Development of anticancer therapies with agents with high with little or no toxicities to normal cells is inevitable. TLR agonists have been extensively known to exert immunomodulatory effects as a standalone therapeutic agent or in boosting therapeutic anticancer response(s) when combined with other treatment regimes against cancer [28,31,32,42]. However, activation of TLRs has been known to exert both antitumor and protumor response(s) in different types of cancer. 
Different cancer cells have been reported to express different TLRs. On stimulation, TLR3 and TLR5 on cancer cells generally stimulate antitumor response, whereas TLR4, -7, -8, and -9 primarily induce protumor effects [20]. Alteration in immune stimulation and immunosuppressive characteristics of the tumor microenvironment are the deciding factors for the desired outcome of the cancer therapy. Activation of different TLRs in cancer cells may play conflicting roles and are summarized in Table 1. Moreover, TLR agonists mediated induction of cytokines or / costimulatory molecules on APCs (or tumor cells) will be an influential factor for the antitumor or protumor response. Figure 3 describes the points to be considered while exploiting a specific TLR agonist against tumor cells. $\mathrm{eT}_{\text {reg }}$ cells numbers is crucial if we need to provide immunomodulatory treatments that target malignancy without severe adverse events [26].

In this review, we summarized the importance of TLRs and their agonists in tumor biology and their exploitation as a standalone or combination therapeutic for anticancer therapy. It is evident that, activation of same TLR on one tumor type might induce cell death, and on a different tumor could exert protumor effects. Selection of TLR agonists against cancer must be done on the basis of TLR expression profile of tumor cells and resulting tumor response within a specific cancer type [20].

\section{References}

1. Singh VK, Ducey EJ, Fatanmi 00, Singh PK, Brown DS (2012) CBLB613: a TLR 2/6 agonist, natural lipopeptide of Mycoplasma arginini, as a novel radiation countermeasure. Radiat Res 177(5): 628-642.

2. Andersen JM, Al-Khairy D, Ingalls RR (2006) Innate immunity at the mucosal surface: role of toll-like receptor 3 and toll-like receptor 9 in cervical epithelial cell responses to microbial pathogens. Biol Reprod 74(5): 824-831.

3. Pradhan VD, Das S, Surve P, Ghosh K (2012) Toll-like receptors in autoimmunity with special reference to systemic lupus erythematosus. Indian J Hum Genet 18(2): 155-160.

4. Sanguri S, Gupta D, Singh T, Singh AK (2016) Biodistribution and scintigraphic evaluation of $99 \mathrm{mTc}-M a n n a n$ complex. Discoveries 4(3): e65.

5. Tipping PG (2006) Toll-like receptors: the interface between innate and adaptive immunity. J Am Soc Nephrol 17(7): 1769-1771.

6. Singh VK, Pollard HB (2015) Patents for Toll-like receptor ligands as radiation countermeasures for acute radiation syndrome. Expert Opin Ther Pat 25(10): 1085-1092.

7. Kawai T, Akira S (2006) TLR signaling. Cell Death Differ 13(5): 816825 .

8. Chua BY, Zeng W, Jackson DC (2008) Synthesis of toll-like receptor-2 targeting lipopeptides as self-adjuvanting vaccines. Methods Mol Biol 494: 247-261.

9. Pfirschke C, Garris C, Pittet MJ (2015) Common TLR5 mutations control cancer progression. Cancer Cell 27(1): 1-3.

10. Rutkowski MR, Stephen TL, Svoronos N, Allegrezza MJ, Tesone AJ, et al. (2015) Microbially driven TLR5-dependent signaling governs distal malignant progression through tumor-promoting inflammation. Cancer Cell 27(1): 27-40.

11. Krieg AM, Vollmer J (2007) Toll-like receptors 7, 8, and 9: linking innate immunity to autoimmunity. Immunol Rev 220: 251-269.
12. Agrawal S, Kandimalla ER (2007) Synthetic agonists of Toll-like receptors 7, 8 and 9. Biochem Soc Trans 35(Pt 6): 1461-1467.

13. Hoebe K, Janssen E, Beutler B (2004) The interface between innate and adaptive immunity. Nat Immunol 5(10): 971-974.

14. Husebye H, Halaas $\emptyset$, Stenmark H, Tunheim G, Sandanger $\emptyset$, et al. (2006) Endocytic pathways regulate Toll-like receptor 4 signaling and link innate and adaptive immunity. EMBO J 25(4): 683-692.

15. Kabelitz D (2007) Expression and function of Toll-like receptors in T lymphocytes. Curr Opin Immunol 19(1): 39-45.

16. Kanzler H, Barrat FJ, Hessel EM, Coffman RL (2007) Therapeutic targeting of innate immunity with Toll-like receptor agonists and antagonists. Nat Med 13(5): 552-559.

17. Huhta H, Helminen O, Kauppila JH, Salo T, Porvari K, et al. (2016) The Expression of Toll-like Receptors in Normal Human and Murine Gastrointestinal Organs and the Effect of Microbiome and Cancer. J Histochem Cytochem 64(8): 470-482.

18. Yoon SI, Kurnasov O, Natarajan V, Hong M, Gudkov AV, et al. (2012) Structural basis of TLR5-flagellin recognition and signaling. Science 335(6070): 859-864

19. Alderton GK (2012) Signalling: New roles for TLR2. Nat Rev Immunol 12(12): 810-811.

20. Kaczanowska S, Joseph AM, Davila E (2013) TLR agonists: our best frenemy in cancer immunotherapy. J Leukoc Biol 93(6): 847-863.

21. Lee CC, Avalos AM, Ploegh HL (2012) Accessory molecules for Toll-like receptors and their function. Nat Rev Immunol 12(3): 168-179.

22. Dadi S, Chhangawala S, Whitlock BM, Franklin RA, Luo CT, et al. (2016) Cancer Immunosurveillance by Tissue-Resident Innate Lymphoid Cells and Innate-like T Cells. Cell 164(3): 365-377.

23. Gajewski TF, Schreiber H, Fu YX (2013) Innate and adaptive immune cells in the tumor microenvironment. Nat Immunol 14(10): 10141022.

24. Pradere JP, Dapito DH, Schwabe RF (2014) The Yin and Yang of Toll-like receptors in cancer. Oncogene 33(27): 3485-3495.

25. Proença MA, de Oliveira JG, Cadamuro AC, Succi M, Netinho JG, et al. (2015) TLR2 and TLR4 polymorphisms influence mRNA and protein expression in colorectal cancer. World J Gastroenterol 21(25): 77307741.

26. Ureshino H, Shindo T, Nishikawa H, Watanabe N, Watanabe E, et al. (2016) Effector Regulatory T Cells Reflect the Equilibrium between Antitumor Immunity and Autoimmunity in Adult T-cell Leukemia. Cancer Immunol Res 4(8): 644-649.

27. Zhang Y, Luo F, Cai Y, Liu N, Wang L, et al. (2011) TLR1/TLR2 agonist induces tumor regression by reciprocal modulation of effector and regulatory T cells. J Immunol 186(4): 1963-1969.

28. Vacchelli E, Vitale I, Tartour E, Eggermont A, Sautès-Fridman C, et al. (2013) Trial Watch: Anticancer radioimmunotherapy. Oncoimmunology 2(9): e25595.

29. Northfelt DW, Ramanathan RK, Cohen PA, Von Hoff DD, Weiss GJ, et al. (2014) A phase I dose-finding study of the novel Toll-like receptor 8 agonist VTX-2337 in adult subjects with advanced solid tumors or lymphoma. Clin Cancer Res 20(14): 3683-3691.

30. Dietsch GN, Randall TD, Gottardo R, Northfelt DW, Ramanathan RK, et al. (2015) Late-Stage Cancer Patients Remain Highly Responsive to Immune Activation by the Selective TLR8 Agonist Motolimod (VTX2337). Clin Cancer Res 21(24): 5445-5452.

31. Vacchelli E, Eggermont A, Sautès-Fridman C, Galon J, Zitvogel L (2013) Trial Watch: Toll-like receptor agonists for cancer therapy. Oncoimmunology 2(8): e25238. 
32. Gearing AJ (2007) Targeting toll-like receptors for drug development: a summary of commercial approaches. Immunol Cell Biol 85(6): 490494

33. Garay RP, Viens P, Bauer J, Normier G, Bardou M, et al. (2007) Cancer relapse under chemotherapy: why TLR2/4 receptor agonists can help. Eur J Pharmacol 563(1-3): 1-17.

34. Azzam EI, Jay-Gerin JP, Pain D (2012) Ionizing radiation-induced metabolic oxidative stress and prolonged cell injury. Cancer Lett 327(1-2): 48-60.

35. Kamiya K, Ozasa K, Akiba S, Niwa O, Kodama K, et al. (2015) Long-term effects of radiation exposure on health. Lancet 386(9992): 469-478.

36. Orrenius S, Gogvadze V, Zhivotovsky B (2007) Mitochondrial oxidative stress: implications for cell death. Annu Rev Pharmacol Toxicol 47 143-183.

37. Cordeiro RM (2014) Reactive oxygen species at phospholipid bilayers: distribution, mobility and permeation. Biochim Biophys Acta 1838(1 Pt B): 438-444.

38. Gulbahar O, Aricioglu A, Akmansu M, Turkozer Z (2009) Effects of radiation on protein oxidation and lipid peroxidation in the brain tissue. Transplant Proc 41(10): 4394-4396.
39. Burdelya LG, Krivokrysenko VI, Tallant TC, Strom E, Gleiberman AS, et al. (2008) An agonist of toll-like receptor 5 has radioprotective activity in mouse and primate models. Science 320(5873): 226-230.

40. Singh V, Gupta D, Arora R (2015) NF- $\kappa B$ as a key player in regulation of cellular radiation responses and identification of radiation countermeasures. Discoveries 3(5): 1-22.

41. Zhang H, Liu L, Yu D, Kandimalla ER, Sun HB (2012) An in situ autologous tumor vaccination with combined radiation therapy and TLR9 agonist therapy. PLoS One 7(5): e38111.

42. Iribarren K, Bloy N, Buqué A, Cremer I, Eggermont A, et al. (2016) Trial Watch: Immunostimulation with Toll-like receptor agonists in cancer therapy. Oncoimmunology 5(3): e1088631.

43. Aurelian L (2016) Oncolytic viruses as immunotherapy: progress and remaining challenges. Onco Targets Ther 9: 2627-2637.

44. Temizoz B, Kuroda E, Ishii KJ (2016) Vaccine adjuvants as potential cancer immunotherapeutics. Int Immunol 28(7): 329-338.

\section{Your next submission with Juniper Publishers} will reach you the below assets

- Quality Editorial service

- Swift Peer Review

- Reprints availability

- E-prints Service

- Manuscript Podcast for convenient understanding

- Global attainment for your research

- Manuscript accessibility in different formats ( Pdf, E-pub, Full Text, Audio)

- Unceasing customer service

Track the below URL for one-step submission https://juniperpublishers.com/online-submission.php 